\begin{tabular}{|c|c|}
\hline Title & Model Reduction of Three-Dimensional Eddy Current Problems Based on the Method of Snapshots \\
\hline Author(s) & Sato, Y uki; Igarashi, Hajime \\
\hline Citation & $\begin{array}{l}\text { IEEE T ransactions On Magnetics, 49(5), 1697-1700 } \\
\text { https://doi.org/10.1109// MA G.2013.2241412 }\end{array}$ \\
\hline Issue Date & $2013-05$ \\
\hline Doc URL & http://hdl.handle.net/2115/53100 \\
\hline Rights & $\begin{array}{l}\text { (c) } 2013 \text { IEEE. Personal use of this material is permitted. Permission from IEEE must be obtained for all other uses, in } \\
\text { any current or future media, including reprinting/republishing this material for advertising or promotional purposes, } \\
\text { creating new collective works, for resale or redistribution to servers or lists, or reuse of any copyrighted component of } \\
\text { this work in other works. }\end{array}$ \\
\hline Type & article (author version) \\
\hline File Information & Model Reduction of Three Dimensional Eddy Current Priblems Based on the Method of Snapshots.pdf \\
\hline
\end{tabular}

Instructions for use 


\title{
Model Reduction of Three Dimensional Eddy Current Problems Based on the Method of Snapshots
}

\author{
Yuki Sato and Hajime Igarashi \\ Graduate School of Information Science and Technology, Hokkaido University, 060-0814 Sapporo, Japan
}

\begin{abstract}
The model reduction based on the method of snapshots is applied to the finite element analysis of three dimensional transient eddy current problems. It is known that accuracy of the reduced model highly depends on the number of snapshots. In this paper, we introduce a novel method which determines the adequate number of snapshots automatically. It is shown that the computational time can be reduced when using the model reduction based on the present method.
\end{abstract}

Index Terms-Model order reduction, method of snapshots, proper orthogonal decomposition, finite element method, eddy current problem.

\section{INTRODUCTION}

$\mathrm{F}$ IITE ELEMENT METHOD (FEM) has widely been used for electromagnetic field analysis of electric machines and devices such as motors and transformers. Three dimensional FE analysis is still time consuming although computer performance has been significantly improving. In particular, heavy computational burden in the three dimensional FE analysis causes serious problems when it is applied to optimizations [1-3]. It is, therefore, strongly required that the computational time for the FE analysis is reduced. The model reduction method is one of the promising methods to reduce the computational time. For the linear problems, the reduction methods based on Padè approximation with the Lanczos processes and Krylov subspace projection have been proposed [4], [5]. However, it seems uneasy to extend these methods to non-linear problems. On the other hand, the method of snapshots based on principal component analysis has been shown effective for the non-linear problems [6-11]. This method is also called the proper orthogonal decomposition [6].

In the model reduction based on the method of snapshot, the variance-covariance matrix is constructed from the independent vectors which express field distributions called snapshots. The basis vectors for the reduced model are then obtained from the eigenvectors of the variance-covariance matrix. It is shown that the model reduction based on method of snapshots is effective for fluid analysis [7], [8] and for quasi-static electromagnetic analysis in two dimensions [9], [10]. This method has also been shown effective for three dimensional quasi-static electromagnetic problems [11].

It has been pointed out in [11] that accuracy of this method highly depends on the number of snapshots. However, it has been unclear how to determine the adequate number of snapshots. In this paper, we introduce a novel method to determine the number of snapshots automatically.

\section{TIME-DOMAIN FINITE ELEMENT METHOD}

The governing equations for eddy current problems derived from Maxwell's equations are expressed as

Manuscript received January 8, 2013. Corresponding author: Hajime Igarashi (e-mail: igarashi@ssi.ist.hokudai.ac.jp).

Digital Object Identifier inserted by IEEE

$$
\begin{aligned}
& \operatorname{rot} v \operatorname{rot} \boldsymbol{A}+\sigma\left(\frac{\partial \boldsymbol{A}}{\partial t}+\operatorname{grad} \frac{\partial \varphi}{\partial t}\right)=\boldsymbol{J}, \\
& \operatorname{div} \sigma\left(\frac{\partial \boldsymbol{A}}{\partial t}+\operatorname{grad} \frac{\partial \varphi}{\partial t}\right)=0,
\end{aligned}
$$

where $\boldsymbol{A} \varphi, v, \sigma$ and $\boldsymbol{J}$ are the vector potential, scalar potential, magnetic resistivity, conductivity and current density. By applying the weighted residual method and the Galerkin method to eqs. (1) and (2), we can obtain the FE equation

$$
\left[\begin{array}{ll}
K & 0 \\
0 & 0
\end{array}\right]\left[\begin{array}{l}
\boldsymbol{a} \\
\varphi
\end{array}\right]+\frac{\mathrm{d}}{\mathrm{d} t}\left[\begin{array}{cc}
\mathrm{N} & \mathrm{S} \\
\mathrm{S}^{\mathrm{t}} & \mathrm{M}
\end{array}\right]\left[\begin{array}{l}
\boldsymbol{a} \\
\varphi
\end{array}\right]=\left[\begin{array}{l}
\boldsymbol{b} \\
0
\end{array}\right],
$$

where

$K_{i j}=\int_{V} \operatorname{rot} N_{i} \cdot \operatorname{rot} N_{j} \mathrm{~d} V$,

$N_{i j}=\int_{V} \sigma N_{i} \cdot N_{j} \mathrm{~d} V$,

$S_{i j}=\int_{V} \sigma \mathbf{N}_{i} \cdot \operatorname{grad} N_{j} \mathrm{~d} V$,

$M_{i j}=\int_{V} \sigma \operatorname{grad} N_{i} \cdot \operatorname{grad} N_{j} \mathrm{~d} V$,

$b_{i}=\int_{V} N_{i} \cdot J \mathrm{~d} V$,

and $N_{i}$ and $N_{j}$ are vector and scalar interpolation functions, respectively. The unknowns $\boldsymbol{a} \in R^{e}$ and $\varphi \in R^{n}$ in eq. (3) are assigned to the edges and nodes of the FE mesh, and the matrices in eq. (3) are $m \times m$, where $m=e+n$. The finite difference scheme in time is applied to eq. (3) to obtain

$$
\begin{aligned}
& \left(\frac{1}{\Delta t}\left[\begin{array}{cc}
\mathrm{N} & \mathrm{S} \\
\mathrm{S}^{\mathrm{t}} & \mathrm{M}
\end{array}\right]+\theta\left[\begin{array}{cc}
\mathrm{K} & 0 \\
0 & 0
\end{array}\right]\right) \boldsymbol{x}^{k}= \\
& \left(\frac{1}{\Delta t}\left[\begin{array}{cc}
\mathrm{N} & \mathrm{S} \\
\mathrm{S}^{\mathrm{t}} & \mathrm{M}
\end{array}\right]-(1-\theta)\left[\begin{array}{cc}
\mathrm{K} & 0 \\
0 & 0
\end{array}\right]\right) \boldsymbol{x}^{k-1}+\left[\begin{array}{c}
\theta \boldsymbol{b}^{k}+(1-\theta) \boldsymbol{b}^{k-1} \\
0
\end{array}\right],
\end{aligned}
$$

where $\boldsymbol{x}^{k}=\left[\boldsymbol{a}^{k} \boldsymbol{\varphi}^{k}\right]^{\mathrm{t}}, 0 \leq \theta \leq 1, \Delta t$ is the time step interval and $k$ 
represents time steps. The original solutions can be determined by solving eq. (9) at each time step.

\section{METHOD OF SNAPSHOTS}

\section{A. Construction of basis vectors}

In order to reduce the computational time, we apply the reduction method based on the method of snapshots to eq. (9). To obtain the snapshots, we solve eq. (9) to obtain the transient solutions $\boldsymbol{x}^{k}$, where $0 \leq k \leq s$, and $s$ represents the number of snapshots $(m>>s)$. Then, the matrix $\mathrm{X} \in R^{m \times s}$ is constructed from $\boldsymbol{x}^{k}$ as

$\mathrm{X}=\left[\begin{array}{llll}x^{1}-\boldsymbol{\mu} & \boldsymbol{x}^{2}-\boldsymbol{\mu} & \cdots & \boldsymbol{x}^{s}-\boldsymbol{\mu}\end{array}\right]$

where $\boldsymbol{\mu}$ is the mean vector of $\boldsymbol{x}^{k}$. Applying the singular value decomposition to $\mathrm{X}$, we obtain

$$
\mathrm{X}=\mathrm{U} \Sigma \mathrm{V}^{\mathrm{t}}=\sigma_{1} \boldsymbol{u}_{1} \boldsymbol{v}_{1}^{t}+\sigma_{2} \boldsymbol{u}_{2} \boldsymbol{v}_{2}^{t}+\cdots+\sigma_{s} \boldsymbol{u}_{s} \boldsymbol{v}_{s}^{t},
$$

where $\boldsymbol{u}_{i}$ and $\boldsymbol{v}_{i}$ are the eigenvectors of the variance-covariance matrix $\mathrm{XX}^{\mathrm{t}}$ and $\mathrm{X}^{\mathrm{t}} \mathrm{X}$, respectively and $\sigma_{i}, i=1,2, \ldots, s$ are the singular values which correspond to the square root of eigenvalues of $\mathrm{XX}^{\mathrm{t}}$. The dominant eigenvectors $\boldsymbol{u}_{i}$ of $\mathrm{XX}^{\mathrm{t}}$ are chosen as the basis vectors for the reduced system. The transfer matrix $\mathrm{W}$ is constructed from $\boldsymbol{u}_{i}$ as follows:

$$
\mathrm{W}=\left[\begin{array}{llll}
\boldsymbol{u}_{1} & \boldsymbol{u}_{2} & \cdots & \boldsymbol{u}_{s}
\end{array}\right],
$$

where $\mathrm{W} \in R^{m \times s}$. The original unknown vector $\boldsymbol{x}^{k}$ can be expressed by the linear combination of $\boldsymbol{u}_{i}$, that is,

$$
\boldsymbol{x}^{k}=\mathbf{W} \boldsymbol{y}^{k},
$$

where $\boldsymbol{y}^{k} \in R^{s}$ is the reduced unknown vector.

\section{B. Formulation for linear problems}

Equation (9) is here written in the form $A \boldsymbol{x}^{k}=\boldsymbol{b}^{k}$ for simplicity to which eq. (13) is substituted to obtain the reduced equation

$$
\mathrm{W}^{\mathrm{t}} \mathrm{AW} \boldsymbol{y}^{k}=\mathrm{W}^{\mathrm{t}} \boldsymbol{b} \text {. }
$$

Because the reduced coefficient matrix $\mathrm{W}^{\mathrm{t}} \mathrm{AW}$ is $s \times s$, eq. (14) can be solved much faster than eq. (9).

\section{Formulation for nonlinear problems}

In the nonlinear problem, the magnetic reluctivity in the magnetic material varies depending on the magnetic flux density. Hence, eq. (9) can be expressed as

$$
\mathrm{A}(v(\boldsymbol{a})) \boldsymbol{x}^{k}=\boldsymbol{b}^{k}
$$

By substituting eq. (13) into eq. (15), we obtain
$\mathrm{W}^{\mathrm{t}} \mathrm{A}(v(\boldsymbol{y})) \mathrm{W} \boldsymbol{y}^{k}=\mathrm{W}^{\mathrm{t}} \boldsymbol{b}^{k}$.

By applying the Newton-Raphson method to eq. (16), we obtain the linearized equation given by

$\frac{\partial \boldsymbol{g}^{\prime}}{\partial \boldsymbol{y}} \delta \boldsymbol{y}=-\boldsymbol{g}^{\prime}$,

where $\boldsymbol{g}^{\prime}=\mathrm{W}^{\mathrm{t}} \boldsymbol{b}^{k}-\mathrm{W}^{\mathrm{t}} \mathrm{A}(v(\boldsymbol{y})) \mathrm{W}^{k}$. The coefficient matrix in eq. (17) can be rewritten as

$\frac{\partial \boldsymbol{g}^{\prime}}{\partial \boldsymbol{y}}=\mathrm{W}^{\mathrm{t}} \frac{\partial \boldsymbol{g}}{\partial \boldsymbol{x}} \mathrm{W}$,

where $\boldsymbol{g}=\boldsymbol{b}^{k}-\mathrm{A} \boldsymbol{x}^{k}$. Consequently, the reduced equation for nonlinear problem is expressed as

$\mathrm{W}^{\mathrm{t}} \frac{\partial \boldsymbol{g}}{\partial \boldsymbol{x}} \mathrm{W} \delta \boldsymbol{y}=-\boldsymbol{g}^{\prime}$

\section{Automatic determination of snapshot numbers}

As mentioned in section I, accuracy of the present method highly depends on $s$ [11]; if we take a too small value for $s$ resultant accuracy is expected to be low. On the other hand, if we take a too large value for $s$, the resultant accuracy would be sufficiently high, but simultaneously, computational time becomes long. Moreover, optimal value for $s$ depends on the problems. In the followings, we introduce an effective method which can determine the number of snapshots $s$ automatically.

The error at step $k$ is defined by

$e_{k}=\sqrt{\frac{\sum_{i}\left|\boldsymbol{B}_{i}-\boldsymbol{B}_{i}^{\text {red }}\right|^{2}}{\sum_{i}\left|\boldsymbol{B}_{i}\right|^{2}}} \times 100(\%)$,

where $\boldsymbol{B}_{i}$ is the original magnetic flux density and $\boldsymbol{B}_{i}^{\text {red }}$ is the magnetic flux density given by eq. (14) or eq. (19). It is expected that $e_{s}$ is relatively small because the basis vectors are constructed from the snapshots by this time step. On the other hand, $e_{s+1}$ would be larger than $e_{s}$ if $s$ is not sufficiently large. To characterize the sudden change in the error at the time steps $s$ and $s+1$, we introduce the ratio defined by

$\gamma \equiv \frac{e_{s+1}}{e_{s}}$

If $\gamma$ is smaller than a certain value, $s$ is judged to be sufficiently large, and the reduced eq. (14) or (19) is solved until steady state. If this condition is not satisfied, the number of snapshots $s$ is increased and the reduced equation is newly constructed. The algorithm for the present method is summarized as follows: 


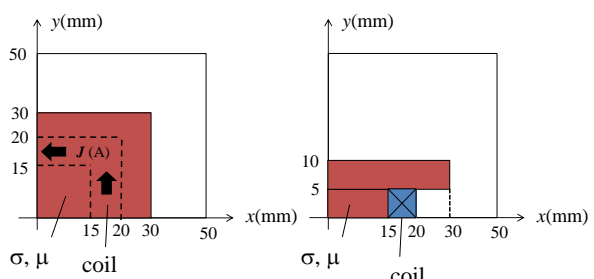

Fig. 1. Model $1\left(\boldsymbol{J}=1 \times 10^{7} \mathrm{~A} / \mathrm{m}^{2}, \sigma=0.5 \times 10^{7} \mathrm{~S} / \mathrm{m}\right)$

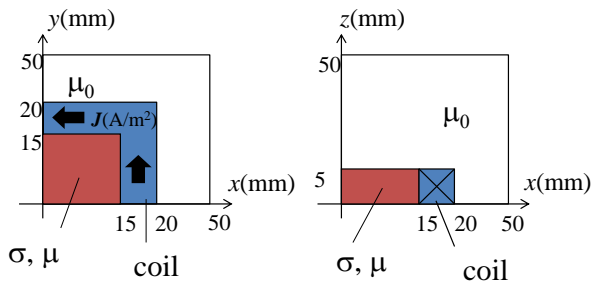

Fig. 2. Model $2\left(\boldsymbol{J}=1 \times 10^{7} \mathrm{~A} / \mathrm{m}^{2}, \sigma=0.5 \times 10^{7} \mathrm{~S} / \mathrm{m}\right)$
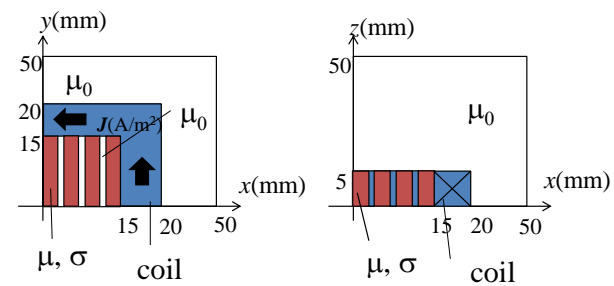

Fig. 3 :Model $3\left(\boldsymbol{J}=2 \times 10^{6} \mathrm{~A} / \mathrm{m}^{2}, \sigma=0.5 \times 10^{7} \mathrm{~S} / \mathrm{m}\right)$

(a) The initial value is set for $s$.

(b) Equation (9) is solved to obtain the original solutions $\boldsymbol{x}^{k}$ until time step $s+1$.

(c) From $\boldsymbol{x}^{k}, k=1,2 \ldots, s$, we construct $\mathrm{X}$, whose singular values are computed to obtain the reduced equations (14) or (19).

(d) Equations (14) or (19) are solved until time step $s+1$.

(e) If $\gamma$, computed from (21), is larger than a threshold, $s \leftarrow s+\Delta s$, and return to (b). Otherwise, the reduced equation is solved until convergence.

\section{NUMERICAL RESULT}

The numerical models in Figs. 1-3 are analyzed by the present method. The domain is discretized by the hexahedral elements which are composed of 125000 nodes, 117649 elements, 367500 edges. The conductivity is $0.5 \times 10^{7} \mathrm{~S} / \mathrm{m}$. The driving frequency $f$ is set to $50 \mathrm{~Hz}$ and $\Delta t=5 \times 10^{-4} \mathrm{sec}$. The initial states are assumed to be zero fields. If we have a good approximation for the steady states, it would be suitable for the initial state.

\section{A. Error Variation}

The error $e_{k}$ is plotted against time steps for Model 1 in Fig. 4 where $\mu_{\mathrm{r}}$ is set to 100 . We can see in Fig.4 that $e_{k}$ is relatively small until $k=s$, and there are sudden increases in $e_{k}$ at $k=s+1$, as expected, especially when $s$ is small. When $s=20$, the increase from $k=s$ to $s+1$ becomes small. These results suggest that $\gamma$ would be a good measure for the accuracy in the reduced model constructed from the snapshots until $s$ steps. In the following computations, the threshold for $\gamma$ is set to 10 and 100 in the linear and nonlinear problems, respectively. These

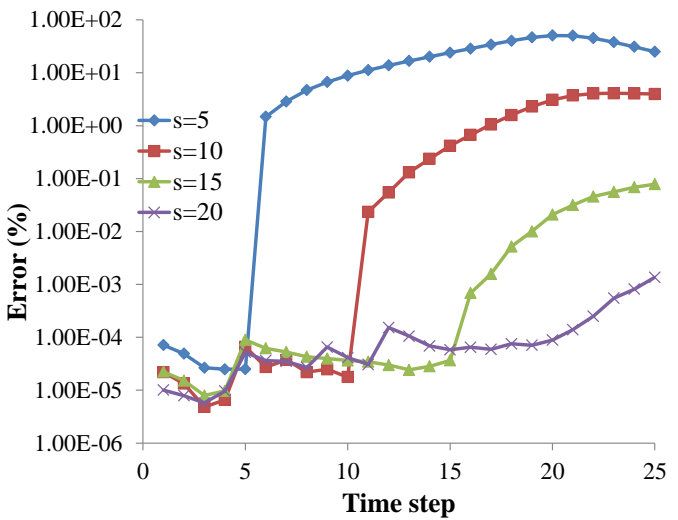

Fig. 4. Error variation with respect to time.

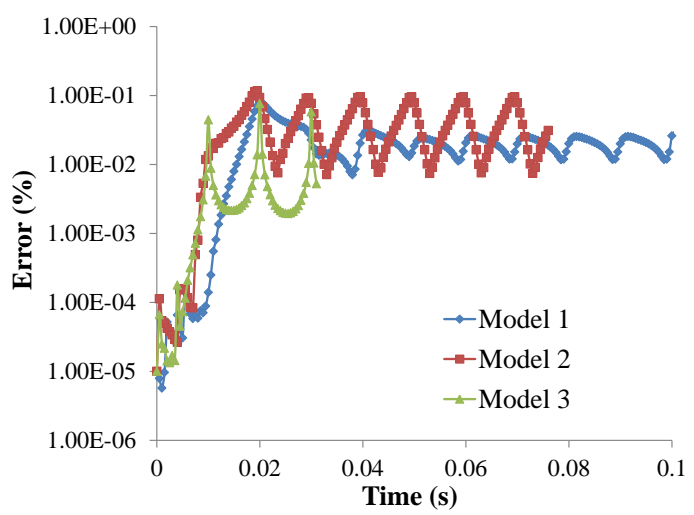

Fig. 5. Error variation with respect to time until steady state.

TABLE I

REDUCTION RESULTS FOR LINEAR PROBLEMS

\begin{tabular}{|c|c|c|c|c|c|}
\hline & Number of snapshots & $s=10$ & $s=15$ & $s=20$ & Automatic \\
\hline & Snapshot time & 0.0045 & 0.007 & 0.0095 & ----- \\
\hline \multirow{3}{*}{$\frac{\overline{0}}{\frac{0}{0}}$} & $\gamma=e_{s+1} / e_{s}$ & 1322 & 18.8 & 1.58 & ---- \\
\hline & Error $e_{\text {steady }}(\%)$ & 4.06 & 0.50 & 0.03 & 0.03 \\
\hline & Com. time $(\%)$ & 12.63 & 15.29 & 18.65 & 19.67 \\
\hline \multirow{3}{*}{$\frac{N}{\frac{N}{0}}$} & $\gamma=e_{s+1} / e_{s}$ & 342 & 5.94 & ---- & ---- \\
\hline & Error $e_{\text {steady }}(\%)$ & 1.74 & 0.08 & 0.07 & 0.08 \\
\hline & Com. time $(\%)$ & 20.00 & 26.07 & 30.35 & 27.86 \\
\hline \multirow{3}{*}{$\frac{\frac{m}{0}}{\frac{0}{2}}$} & $\gamma=e_{s+1} / e_{s}$ & 1.50 & ---- & ---- & ---- \\
\hline & Error $e_{\text {steady }}(\%)$ & 0.005 & 0.002 & 0.0003 & 0.005 \\
\hline & Com. time $(\%)$ & 30.22 & 36.43 & 53.63 & 35.89 \\
\hline
\end{tabular}

* The value of $s$ is automatically determined by the present method.

values of $\gamma$ are determined from the results of numerical experiments.

\section{B. Test for Linear Problems}

The present method is applied to the three models in Figs. 1-3 where $\mu_{\mathrm{r}}$ is set to 100 . The results are summarized in Table I, where the computation time is normalized to that of the conventional computations without reduction which are continued until the change in the amplitude is smaller than $0.01 \%$. From Table I, we can find that error ratio $\gamma$ decreases with increase in the snapshot number $s$. Moreover, we find that $e_{\text {steady, }}$ which is the mean error in the steady state, is less than $0.1 \%$ when $s$ is determined by the present method. 


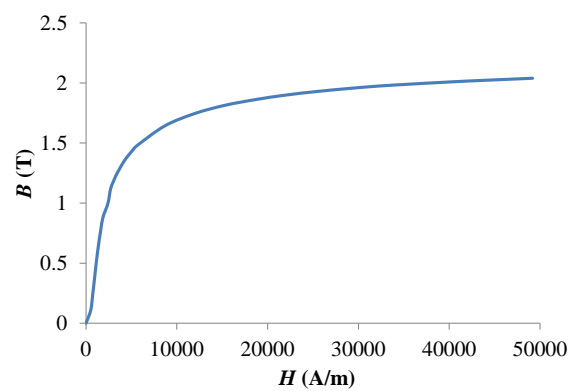

Fig. 6. BH curve

TABLE II

REDUCTION RESULTS FOR NONLINEAR PROBLEMS

\begin{tabular}{|c|c|c|c|c|c|}
\hline \multirow{5}{*}{$\frac{\vec{\nabla}}{\frac{\nabla}{\Sigma}}$} & Number of snapshots & $s=50$ & $s=60$ & $s=70$ & Automatic \\
\hline & Snapshot time & 0.0245 & 0.0295 & 0.0345 & ----- \\
\hline & $\gamma=e_{s+1} / e_{s}$ & 2093 & 1860 & 2.1 & ----- \\
\hline & Error $e_{\text {steady }}(\%)$ & 4.6 & 4.8 & 3.7 & 3.7 \\
\hline & Com. time (\%) & 54.5 & 63.5 & 74.6 & 86.6 \\
\hline \multirow{5}{*}{$\frac{d}{\frac{N}{8}}$} & Number of snapshots & $s=40$ & $s=50$ & $s=60$ & Automatic \\
\hline & Snapshot time & 0.0195 & 0.0245 & 0.0295 & ----- \\
\hline & $\gamma=e_{s+1} / e_{s}$ & 5778 & 3.65 & ----- & ----- \\
\hline & Error $e_{\text {steady }}(\%)$ & 2.2 & 1.8 & 1.2 & 1.8 \\
\hline & Com. time (\%) & 45.9 & 54.4 & 74.4 & 58.2 \\
\hline \multirow{5}{*}{$\frac{\frac{m}{0}}{\frac{\pi}{2}}$} & Number of snapshots & $s=20$ & $s=25$ & $s=30$ & Automatic \\
\hline & Snapshot time & 0.0095 & 0.012 & 0.0145 & ----- \\
\hline & $\gamma=e_{s+1} / e_{s}$ & 7000 & 452.3 & 83.1 & ----- \\
\hline & Error $e_{\text {steady }}(\%)$ & 2.0 & 0.34 & 0.28 & 0.28 \\
\hline & Com. time (\%) & 29.7 & 37.3 & 43.1 & 47.3 \\
\hline
\end{tabular}

Therefore, the present method can determine the adequate number for $s$ automatically. Moreover, the computational time is shorter than that of the conventional method without reduction in all the models.

\section{Test for Nonlinear Problems}

We apply the present method to the models in Figs. 1-3 where we assume that the magnetic material obeys the $\mathrm{BH}$ curve shown in Fig. 6. The results are summarized in Table II, from which we can see that the present method is effective since its computational time is shorter than that of the conventional method. The reduction effect in the computational time for Model 1 becomes weaker than that in the linear model. One of the reasons of this degradation is that the convergence in the Newton-Raphson iteration has relatively slow convergence. Moreover, $e_{\text {steady }}$ is also relatively large for Model 1. Figure 7 shows the error distribution in magnetic material for Model 1 at 182-th step of the reduced model where $e_{k}$ is the maximum. From Fig. 7, we can see that the error is relatively large near the coil. This suggests that the reduced model could not accurately express the localized saturated fields from the snapshots.

\section{CONCLUSION}

In this paper, we have presented a novel method to determine the adequate snapshot number $s$ for the reduction method.

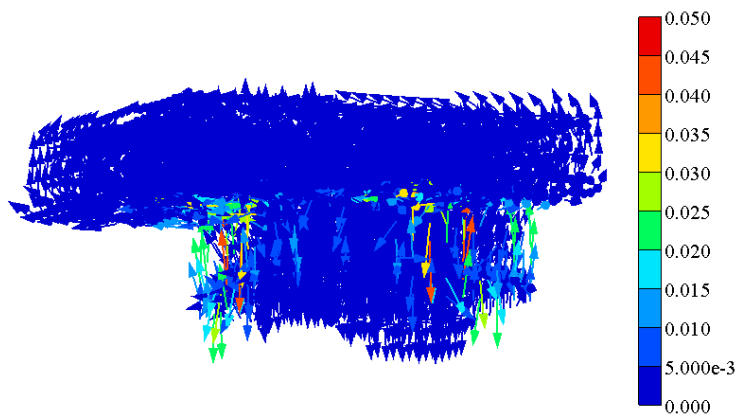

Fig. 7 Error distribution in magnetic material (Model 1). The relatively large errors near the coil would be due to magnetic satulation.

It has been shown that the present method can effectively determine the adequate snapshot number so that the accuracy of the reduction method is satisfactory. Moreover, the computational time for the reduction method based on the present method has been shown to be shorter than that of the conventional method. To improve the accuracy in the reduced system, localized errors due to magnetic saturation should be reduced. This might be possible if the heavily saturated regions are excluded from the domain to which the present method is applied.

\section{REFERENCES}

[1] Y. Watanabe, K. Watanabe and H. Igarashi, "Optimization meander line antenna considering coupling between non-linear circuit and electromagnetic waves for UHF-band RFID," IEEE Trans. Magn., vol. 47, no.5, pp 1506-1509, May 2011.

[2] B. Mirzaeian, M. Moallem, V. Tahani, and C. Lucas, "Multiobjective optimization method based on a genetic algorithm for switched reluctance motor design," IEEE Trans. Magn., vol. 38, no. 3, pp. 15241527, May 2002.

[3] K. Watanabe, F. Campelo, Y. Iijima, K. Kawano, T. Matsuo, T. Mifune, and H. Igarashi, "Optimization of Inductors Using Evolutionary Algorithms and Its Experimental Validation," IEEE Trans. Magn., vol. 46, no. 8, pp. 3393-3396, Aug. 2010.

[4] B. Essakhi, L. Pichon, G. Akoun, "Fast Analysis of a Broad-Band Microwave Rectenna Using 3-D FEM and Pade Approximation," IEEE Trans. Magn, vol. 43, no. 4, pp. 1309-1312, Apr 2007.

[5] R. Börner, O. G. Ernst and K. Spitzer, "Fast 3D simulation of transient electromagnetic fields by model reduction in the frequency domain using Krylov subspace projection," Geophys. J. Int, vol. 173, no. 3, pp. 766-780, June 2008.

[6] G. Kerschen, J. Golinval, A. F. Vakakis and L. A. Bergman, "The Method Proper Orthogonal Decomposition for Dynamical Characterization and Order Reduction of Mechanical Systems: An Overview," Nonlinear Dynamics, vol. 41, no. 1-3, pp. 147-169, Aug. 2005.

[7] P. Krysl, S. Lall, and J.E. Marsden, "Dimensional Model Reduction in Non-Linear Finite Element Dynamics of Solids and Structures," Int. J. Numer. Meth. Engng, vol. 51, pp. 479-504, 2001.

[8] R. A. Handler, K. D. Housiadas and A. N. Beris, "Karhunen-Loeve representations of turbulent channel flows using the method of snapshots," Int. J. Numer. Meth. Fluids, vol 52, pp. 1339-1360, 2006.

[9] S. Rutenkroger, B. Deken, and S. Pekarek, "Reduction of model dimension in nonlinear finite element approximations of electromagnetic systems," in IEEE Workshop on Computers in Power Electronics, Aug. 2004, pp. 20-27.

[10] D. Schmidthäusler and M. Clemens, "Low-Order Electroquasistatic Field Simulations Based on Proper Orthogonal Decomposition," IEEE Trans. Magn., vol. 48, no. 2, pp, 567-570, Feb. 2012.

[11] Y. Sato and H. Igarashi, "On Effectiveness of Model Order Reduction for Computational Electromagnetism," The 15th International IGTE Symposium on Numerical Field Calculation in Electrical Engineering, Abstract, pp, 77, 2012. 